\title{
Pratique artistique et prise en charge de la mémoire : Sofiane Zouggar, artiste et archiviste
}

Rym Khene

\section{Q OpenEdition}

\section{Journals}

Édition électronique

URL : https://journals.openedition.org/coma/5441

DOI : 10.4000/coma.5441

ISSN : 2275-1742

Éditeur

Institut des textes \& manuscrits modernes (ITEM)

Référence électronique

Rym Khene, «Pratique artistique et prise en charge de la mémoire : Sofiane Zouggar, artiste et archiviste », Continents manuscrits [En ligne], 14 | 2020, mis en ligne le 24 mai 2020, consulté le 12 janvier 2023. URL : http://journals.openedition.org/coma/5441 ; DOI : https://doi.org/10.4000/coma. 5441

Ce document a été généré automatiquement le 12 janvier 2023.

\section{(c) (i) () $\Theta$}

Creative Commons - Attribution - Pas d'Utilisation Commerciale - Pas de Modification 4.0 International - CC BY-NC-ND 4.0

https://creativecommons.org/licenses/by-nc-nd/4.0/ 


\title{
Pratique artistique et prise en charge de la mémoire : Sofiane Zouggar, artiste et archiviste
}

\author{
Rym Khene
}

Of course, the 'I' who writes here must also be
thought of as, itself, 'enunciated'. We all write
nd speak from a particular place and time, from
history and a culture which is specific. What we
say is always 'in context', positioned. ${ }^{1}$

1 Mon travail de thèse porte sur la ville d'Alger pendant les années 1990, et pose la question du passage de l'image à la représentation en interrogeant les modalités par lesquelles s'élaborent les représentations d'une ville. Mon choix d'Alger dans les années 1990 est en partie déterminé par certaines continuités qui trouvent leur racine dans la constitution même de la ville dès les débuts de la colonisation, ainsi que par des discontinuités, qui placent Alger au cœur d'une histoire où le passage de l'image à la représentation est le fruit d'évolutions historiques que j'ai choisi de saisir par le prisme commun de la littérature et de la photographie.

2 Lorsque j'ai entrepris mon travail de recherche, et avant de le recentrer sur la ville et les questions spatiales, je me suis interrogée sur le fait qu'on dise de la guerre intérieure ${ }^{2}$ des années 1990 qu'elle est sans images. "Une guerre sans images ${ }^{3}$ » : cette expression, tant de fois entendue pendant plus de deux décennies résonne désormais comme une formule, ou un adage.

3 La guerre intérieure est-elle réellement une guerre sans images ? Cette question semble être un passage obligé lorsque l'on parle - et que l'on part - du contexte algérien. Depuis la guerre d'indépendance, alors que le FLN utilise la photographie comme un des outils de la guerre de libération contre une puissance qui utilise aussi la photographie pour asseoir le pouvoir colonial, la réception des images à l'intérieur aussi bien qu'à l'extérieur du pays interroge, et ces interrogations sont exacerbées par les événements des années 1990, période pendant laquelle est entretenu un mythe 
autour de la visibilité et/ou de l'invisibilité de la guerre, et des images. Ainsi, l'idée fort répandue, qui consiste à insister sur le manque d'images est telle que mon premier réflexe a été de faire appel à ma propre mémoire. Je me suis remémoré ce dont je me souvenais de cette période et je me suis demandé quel était mon rapport à certaines images que j'ai croisées, dans la rue, à la télévision, en photographie, dans un livre. Ce processus de faire défiler des images m'a menée à interroger le rapport à la réalité de certaines d'entre elles ainsi que l'expérience des années 1990 d'où ces images proviennent.

4 J'ai également systématiquement posé cette question, lors des différents entretiens avec les photographes que j'ai rencontrés au long de ma recherche, tout en sachant que cette question était convenue : "Les années 1990 sont-elles sans images? » La réponse a toujours été la même. Sans équivoque. Assurément, il y a des images, un surplus même d'images. Les consciences et les mémoires en sont habitées. Or, ce qui devient apparent au cours des conversations, est que finalement, les images sont souvent orphelines; même si leur auteur est parfois connu, elles se situent dans un espace étroitement lié à l'expérience de la violence, comme son illustration en quelque sorte, qui côtoie une mise en scène, un récit intime de cette violence. Cette expérience particulière de l'image mène alors à se demander quand et comment une image est-elle lue.

5 Si l'on admet qu'il existe bel et bien des images, ce qui ressort des différents entretiens, est le fait que ces images ne sont pas assez interrogées. La photographie reste un domaine négligé dans la culture visuelle algérienne, même si depuis une dizaine d'années environ, il y a une progression certaine de la pratique photographique ainsi que de son étude, à l'université et à travers la pratique artistique.

6 Les travaux de Sofiane Zouggar, artiste algérien né en 1982 et formé à l'école des BeauxArts d'Alger, sont en grande partie ancrés dans la période des années 1990. J'ai découvert son travail et rencontré l'artiste à Alger en 2012 lors d'une exposition collective au Box24, une structure indépendante d'art contemporain, où il présentait une installation vidéo intitulée Time machine, un télescopage d'images des années 1990 et de la guerre de libération ponctuées de scènes de vie, de danse et de pratiques sportives. En 2012, je n'étais pas encore «chercheuse » puisque je m'inscrirai en thèse deux années plus tard, mais je m'étais déplacée à Alger pour faire les photographies de ce qui deviendrait Détours; cette série photographique née d'une absence. Alger est une ville dans laquelle j'ai grandi enfant et vers laquelle je retourne souvent, physiquement ainsi que par la photographie et les livres. En photographiant, j'étais à la recherche de la ville et de ses habitants, je voulais saisir ce que je voyais dans le cadre d'une photographie mais aussi ce qui intervient hors-champ. Cette absence, celle qui trouvait sa source dans le fait d'être partie à l'âge de dix ans était aussi la recherche d'une autre absence, voisine et simultanée, celle d'images des années 1990. L'art et la recherche académique m'ont alors semblé être les chemins d'une quête commune.

7 Memory of Violence de Sofiane Zouggar prend naissance en 2013 et continue à ce jour. Ce projet met en miroir la pratique de l'artiste avec celle du chercheur. En quelques années, Sofiane Zouggar a accumulé une archive considérable constituée de photographies, d'entretiens et de dessins, matériau qu'il ne cesse d'interroger. À travers la mobilisation de ce fonds d'archive, il exhume des photographies pour rendre à la mémoire individuelle et collective des images délaissées de la narration de la guerre intérieure. 
Dans cet article, je me propose d'explorer le projet Memory of Violence, afin d'interroger le geste archivistique de l'artiste dans sa spécificité mais aussi en le rapprochant du geste du chercheur, dans le contexte de la guerre intérieure en Algérie.

\section{La genèse du travail de mémoire : une rencontre photographique « sans nom »}

9 Au début de toute entreprise de recherche ou projet artistique, il y a une intuition. L'artiste et le chercheur ne savent pas, au préalable, ce qu'ils trouveront, mais une lecture, un document, une image vont impulser le travail des années qui vont suivre. Pour Sofiane Zouggar, l'aventure débute avec trois photographies confiées par un jeune homme qui a fait son service militaire à Jijel pendant les années 1990. Lorsqu'il le pouvait, il prenait des photographies avec des appareils jetables qu'il s'était procurés avant de commencer son service militaire de deux ans. Il ne savait pas ce qui l'attendait, et ne soupçonnait pas que ses photographies allaient susciter l'intérêt de l'artiste qui s'en empare comme d'un matériau vivant et complexe, pétri de récits intimes qui vont nourrir le regard et impulser l'entreprise de grande ampleur que va devenir Memory of violence.

10 Un fonds de près de trois cents photographies est alors à sa disposition. D'emblée, Zouggar en extrait trois qui deviendront le triptyque "sans nom» (c'est ainsi que l'artiste légende les photographies d'archive qui ne sont pas les siennes). Dans un souci de protection de l'anonymat de l'auteur des photographies, et certainement par une intuition qui va être la matrice de son travail, Sofiane Zouggar entreprend de découper les figures visibles de deux photographies. En résultent des figures armées qui deviennent alors anonymes. L'aspect quasi fantomatique et l'apparence irréelle de la nouvelle texture proposent instantanément un discours sur l'archive. En effet, l'archive qu'on a pensée inexistante est en réalité silencieuse et invisible jusqu'au jour où elle est exhumée. En partant d'un récit personnel, du souvenir photographique d'un individu, rendre ces figures anonymes contribue aussi à rendre à la photographie sa dimension collective; certains spectateurs, nous confie l'artiste, demanderont même si ces photographies sont issues de la période de la guerre d'indépendance algérienne (1954-1962).

11 Avec ce triptyque, Zouggar ressuscite des images et par ce geste, crée un nouvel espace d'écriture de la mémoire. En modifiant l'archive, l'artiste bouscule la mémoire et ainsi la fait sortir du silence. 
Figures 1 et 2
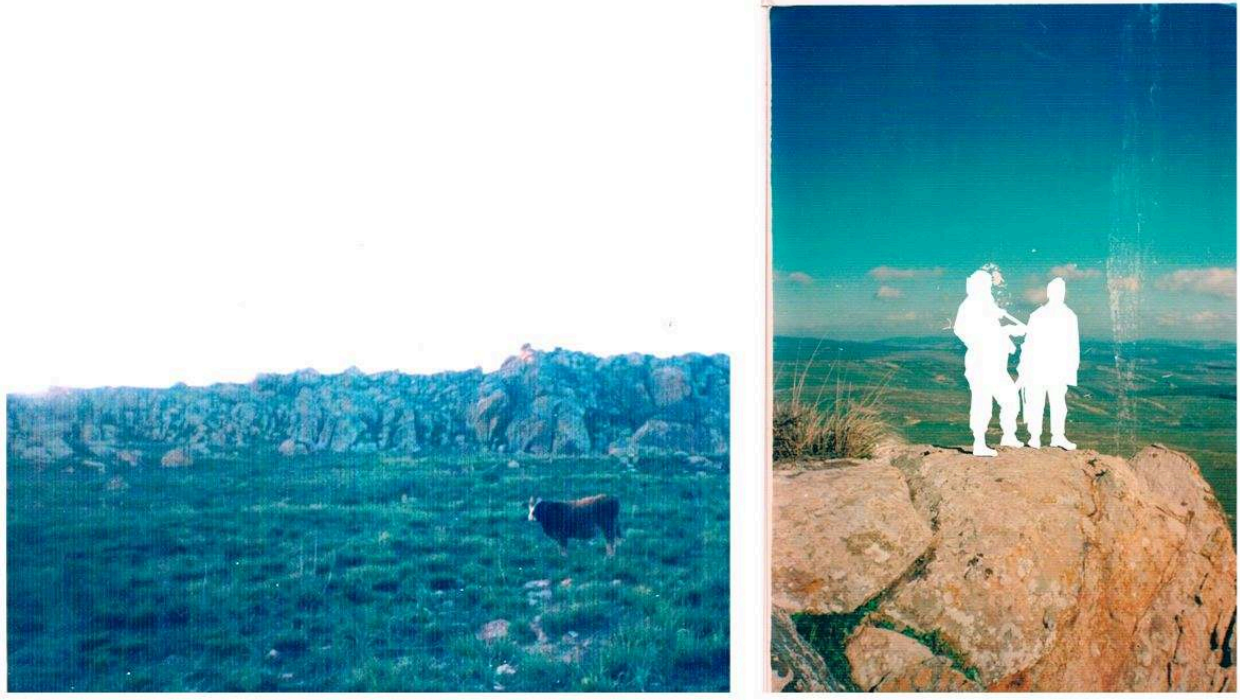

Sofiane Zouggar, les deux premiers tableaux du triptyque « Sans nom ». Impression digitale, de la série The Memory of Violence, $65 \times 43 \mathrm{~cm}, 2014$.

(c) Musée d'Art Moderne et Contemporain d'Alger

\section{Figure 3}

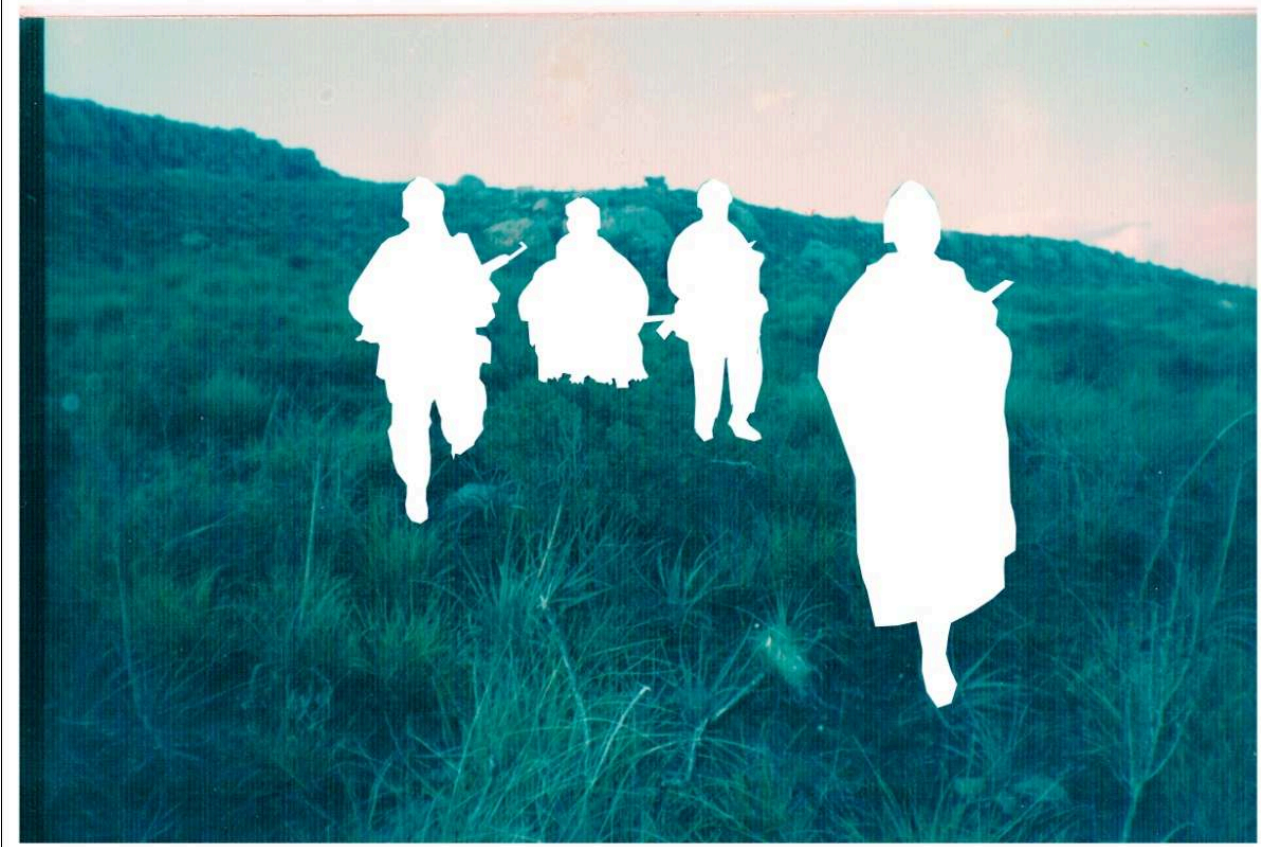

Sofiane Zouggar, troisième tableau du triptyque « Sans nom ». Impression digitale, de la série The Memory of Violence, $65 \times 43 \mathrm{~cm}, 2014$.

(c) Musée d'Art Moderne et Contemporain d'Alger 


\section{In and Out - Tours de surveillance comme « lieux de mémoire »} surveillance a été plusieurs fois évoquée. Aujourd'hui désertées, elles étaient, dans les années 1990, un poste de surveillance des activités terroristes. L'un des témoignages récoltés par Zouggar est celui d'un habitant d'un village proche de Khemis Miliana. L'homme lui raconte qu'aujourd'hui, alors que la guerre est terminée, il ne peut s'empêcher lorsqu'il passe à côté de l'une de ces tours, de penser à ces années, au sentiment de sécurité qu'il éprouvait à la vue de ces tours qui protégeaient le périmètre environnant. Le témoin ne s'est pas attardé sur des souvenirs précis mais cette remarque a mené l'artiste à se demander ce que l'on pouvait ressentir face à ces constructions, aujourd'hui inhabitées, mais qui pour certains, recèlent encore des fantômes; des lieux, même si personne ne les occupe, qui projettent sur les passants, l'impression qu'ils sont surveillés. demande alors quelles sont les histoires de ces tours et ce qu'elles évoquent. Comment aujourd'hui les raconter, les signifier ou leur donner corps? Zouggar a décidé de les photographier. À la manière des passants qui reviennent sur des lieux de mémoire, l'artiste, lui aussi, s'approprie ce mouvement ; sur un temps long, il revisite les tours et les photographie, peut-être pour «faire émerger les virtualités cachées de l'espacetemps ${ }^{4} »$.

rsqu'il accepte l'invitation d'exposer une partie du projet Memory of Violence à New York ${ }^{5}$, Zouggar choisi de mettre en regard trois photographies des tours de surveillance avec la projection d'une quarantaine de photographies qui lui ont été confiées par un ancien terroriste aujourd'hui repenti. En effet, après la première série "sans nom ", Zouggar souhaite explorer l'envers de cette histoire, celle dont on ne parle plus, et que l'on a voulu même réduire au silence par la loi ${ }^{6}$. Il entreprend alors des entretiens avec le repenti qui, en voyant le travail de suppression des figures que Zouggar avait opéré, fait la même chose, à l'aide d'un logiciel informatique, pour protéger son anonymat. Ensuite, il enverra des copies de ces photographies à l'artiste. Avec ce geste, le repenti partage son expérience et crée, lui aussi, une nouvelle archive. 
Figure 4

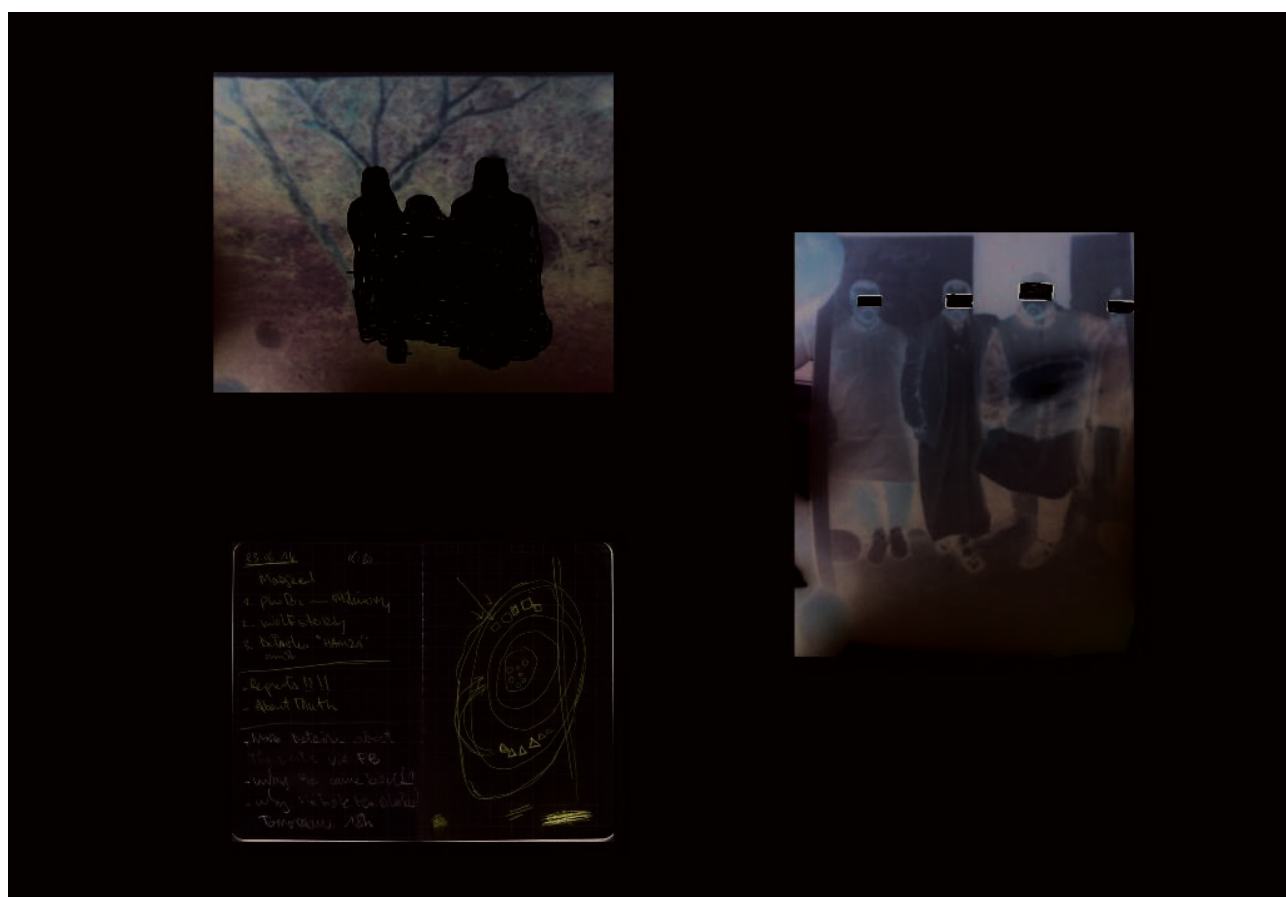

Détail. Memory of Violence de Sofiane Zouggar.

(c) Sofiane Zouggar

Dans le cadre de cette exposition, juxtaposer les photographies des tours de surveillance avec celles du repenti, met en scène le mouvement d'aller-retour des deux protagonistes, à savoir celui de l'artiste qui photographie les constructions et celui du repenti qui, à l'occasion de chaque tombée de neige, retourne dans les montagnes, sur les lieux de ses prises de vues. Télescoper ainsi les regards et les histoires est pour l'artiste, une manière de faire voir les différents volets du projet Memory of Violence jamais exposé en entier, mais, il propose quelque chose de plus subversif, au vu du traitement de l'histoire de cette période.

En effet, confronter les regards et interroger les lieux de mémoire, c'est mettre à mal l'histoire en rendant visible ce que le temps a voulu taire. Placer des photographies de tours de surveillance qui ne surveillent plus, en miroir de photographies prises par un repenti, cristallise un point de tension réel qui prend sa source dans le silence imposé par la loi, celle d'une « réconciliation nationale » qui ne réconcilie ni les victimes ni les bourreaux avec l'histoire.

17 Ainsi, avec le travail de Sofiane Zouggar, des années après la guerre intérieure, les potentialités artistiques - et politiques - de cette période deviennent apparentes et passent dans un premier temps, par l'accumulation d'archives. Ces archives, au parcours parfois aléatoire, vont constituer une mosaïque d'images à inscrire dans un récit collectif. 


\section{Figure 5}

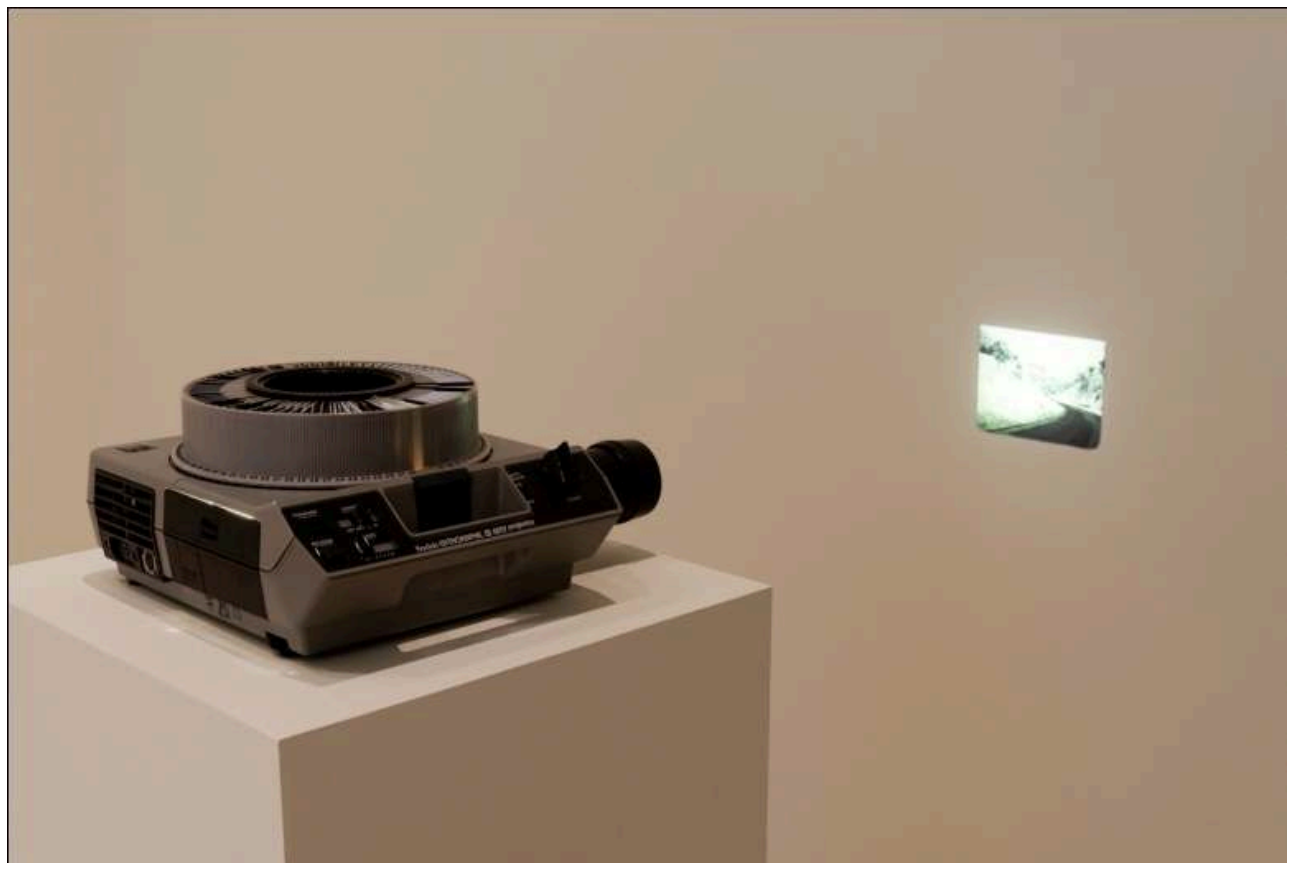

Photo de l'exposition collective Waiting for Omar Gatlato: A Survey of Algerian Contemporary Art, Columbia University, The Wallach Art Gallery, New York (2019).

(c) Sofiane Zouggar

Figure 6

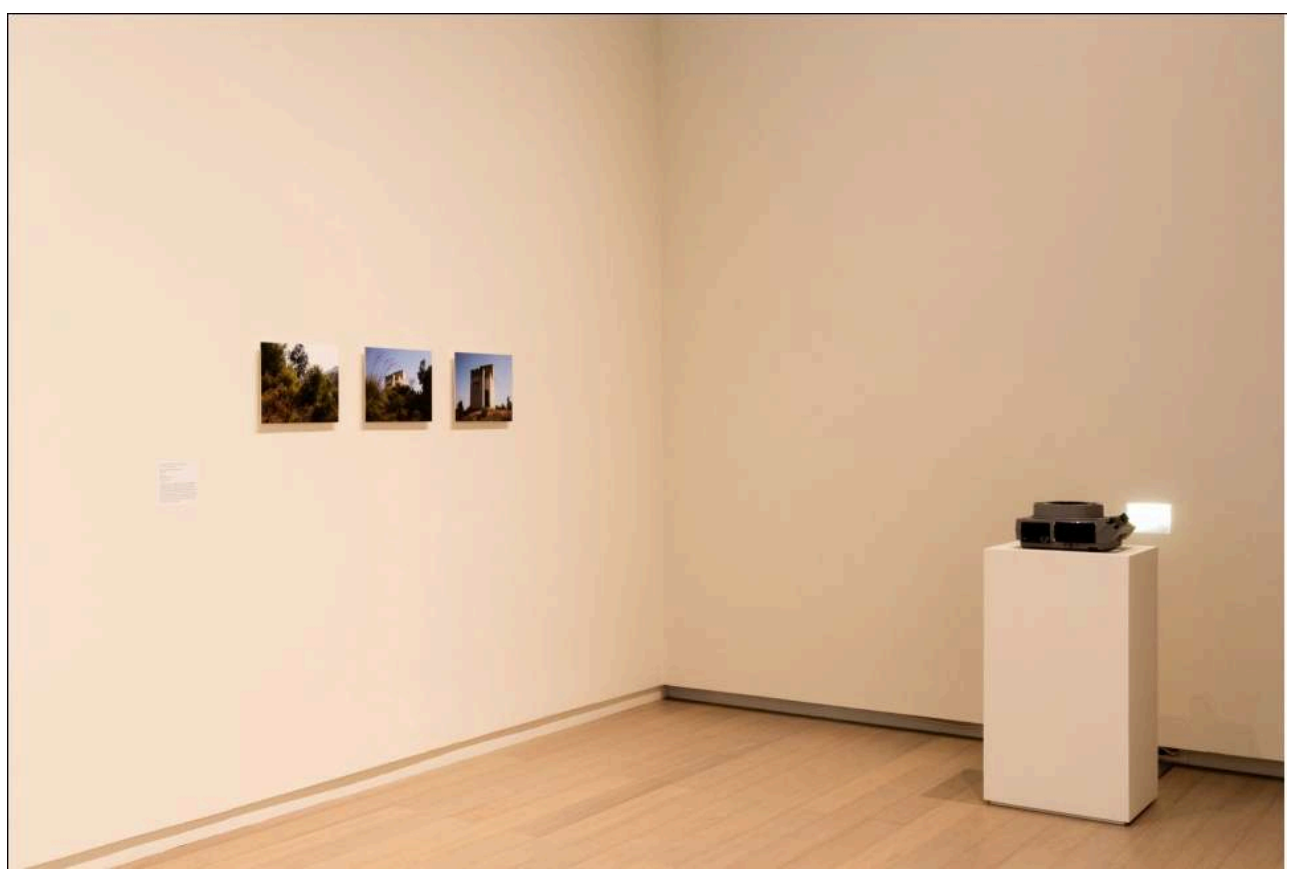

Photo de l'exposition collective Waiting for Omar Gatlato: A Survey of Algerian Contemporary Art, Columbia University, The Wallach Art Gallery, New York (2019).

(c) Sofiane Zouggar 


\section{Mosaïque d'images animées}

18 Un autre volet de Memory of Violence prend la forme d'une installation. Sofiane Zouggar entreprend de rassembler des dizaines d'images qui ont circulé pendant les années 1990. Toutes sortes d'images isolées qui circulent sans récit clair, sans représentation, mais qui sont perçues comme autant de cristallisations de la violence de la guerre intérieure. L'idée, ingénieuse est simple: installer des socles dans de la terre et y poser des écrans qui diffusent en boucle des images des années 1990, comme seule source de lumière. L'objectif : faire pousser des plantes. La question qu'il pose est donc de savoir si l'on peut faire grandir une génération, toute une population en les nourrissant d'images sans qu'elles soient mises en récit. L'expérience était compromise d'avance puisque la lumière émise par les écrans n'était pas assez forte pour réellement faire grandir des plantes mais le geste, symbolique à travers l'installation, permet à l'artiste d'interroger le traitement de la mémoire de la guerre en demandant en filigrane : comment faire sens des images des années 1990 ?

Les photographies ont été en partie récupérées sur Internet, et d'autres sont des photographies de particuliers issues des différentes régions d'Algérie et qui représentent, selon l'artiste, les événements marquants de cette période, tels que l'assassinat du président Mohamed Boudiaf, le massacre de Bentalha, les assassinats de Mostaganem, l'attentat devant le commissariat du boulevard Amirouche... Il ajoute des extraits de documentaires des télévisions française et algérienne, d'embuscades, de terroristes, des photographies de Michael von Graffenried et de Hocine Zaourar, lauréat du prix World Press 1998, pour sa photographie La Madone de Bentalha. Il s'agit d'un ensemble hétéroclite de plus de 90 images, orphelines.

Avec cette installation, Zouggar propose alors un regard sur l'histoire qui par l'utilisation et la réutilisation de photographies diffusées tout au long de cette décennie, interroge la notion d'événement et propose de cartographier l'impact de la violence. Ainsi, Zouggar pose la question du support et de la réutilisation des images, qui n'offrent pas la même lecture selon le lieu où elles sont diffusées, mais propose également une réflexion sur la visualité de la guerre intérieure qui est l'objet d'une série d'opérations discursives ${ }^{7}$ qui sont un moyen de "justifier une autorité qui imaginera l'histoire ${ }^{8} »$. Pour Nicholas Mirzoeff, cette visualisation de l'histoire se situe dans le «droit de voir» (the right to look) qui appartient à ceux qui ont la possibilité d'agencer le visible et le dicible, mais pas seulement. Le droit de voir «n'est pas seulement un assemblage d'images visuelles mais le terrain dans lequel ces assemblages peuvent inscrire une interprétation significative d'un événement donné ${ }^{~}$; c'est aussi cette tension, entre qui regarde et ce que l'on donne à regarder, qui transparait, de façon implicite, dans Memory of Violence. Y a-t-il une image manquante ? Comment avoir une image globale de cette période? Est-ce nécessaire? Pour ceux qui n'ont pas vécu les années 1990 mais qui sont aussi le produit de cette histoire, quelles images constituent cette période pour eux? Toutes ces questions viennent à l'esprit lorsque l'on est confronté au travail de l'artiste qui, au jour d'aujourd'hui, n'a pas encore eu la possibilité d'exposer ce travail en Algérie. L'installation devient elle-même une image qui deviendra archive pour continuer d'interroger la mémoire. 
Figures 7,8 et 9

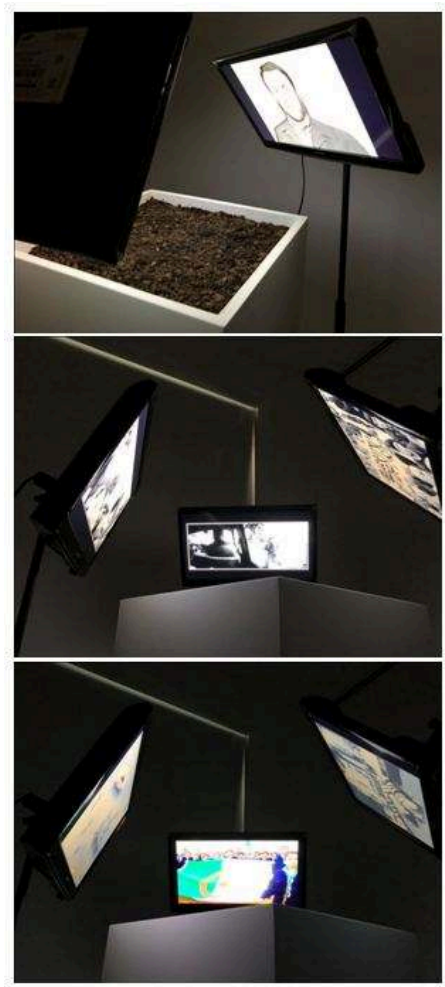

Installation Memory of Violence de Sofiane Zouggar à Sharjah, 2018. Écrans, boîtes en bois, terre et tripodes. Dimensions variables. Installation commissionnée par Sharjah Art Foundation en collaboration avec A.R.I.A (Artist Residency in Algiers).

(C) Sofiane Zouggar

\section{Fabriquer une archive « contre la photographie »}

Pour l'artiste et chercheur Akram Zaatari, aller «contre la photographie, c'est [...] s'appuyer contre l'histoire de la photographie afin de se déplacer, d'aller ailleurs où l'on peut sauver la photographie de son destin..$^{10}$ » Pour ce "faiseur d'image», la pratique photographique permet «de renverser les narrations historiques dominantes » ainsi que de se distancier de la photographie comme document afin de pouvoir la faire dialoguer avec d'autres documents ${ }^{11}$.

Dans sa pratique artistique, Sofiane Zouggar opère ce double mouvement qui devient un déplacement narratif, une démarche qui passe par la fabrique d'archive. Créer sa propre archive, «contre la photographie» parfois, devient alors un processus fondamental pour faire exister, à sa manière et dans un récit personnel, des images qui habitent les imaginaires; les faire dialoguer en les retirant des représentations invariables et durables qui sont proposées. C'est ce qu'il fait avec le triptyque «sans nom ", et reproduit ce déplacement avec le projet d'animation intitulé What remains?, autre volet de Memory of Violence.

23 L'artiste reprend des plans-séquences de documentaires, isole les images qui l'interpellent et les dessine, afin de construire une trame narrative qui serait le reflet de son expérience personnelle des années 1990. Il me dit : «ce film, toujours en cours, est une exploration des images qui restent. J'ai voulu prendre ce que je perçois comme 
essentiel, et cela peut être une silhouette, pour faire mon propre montage, écrire mon histoire de ces images que j'ai vu défiler sous mes yeux pendant des années. » De ces films, il extrait donc des photographies afin, en quelque sorte, de les libérer et de leur donner une nouvelle vie. Près de 2000 dessins permettent à Zouggar de saisir un mouvement qui, tout en prenant sa source dans des images photographiques, l'en éloigne et offre une distance nécessaire pour les lire. Dessiner à la main, prendre le temps de répéter les mouvements, les circonscrire ; voyager entre la photographie et la vidéo, entre des archives mises à nu et des dessins, pour se les approprier davantage, afin de tenter un récit complexe et parfois contradictoire. Sofiane Zouggar s'empare de différents matériaux et navigue entre les supports, "contre la photographie», pour entrevoir « ce qu'il reste».

Figures 10 et 11
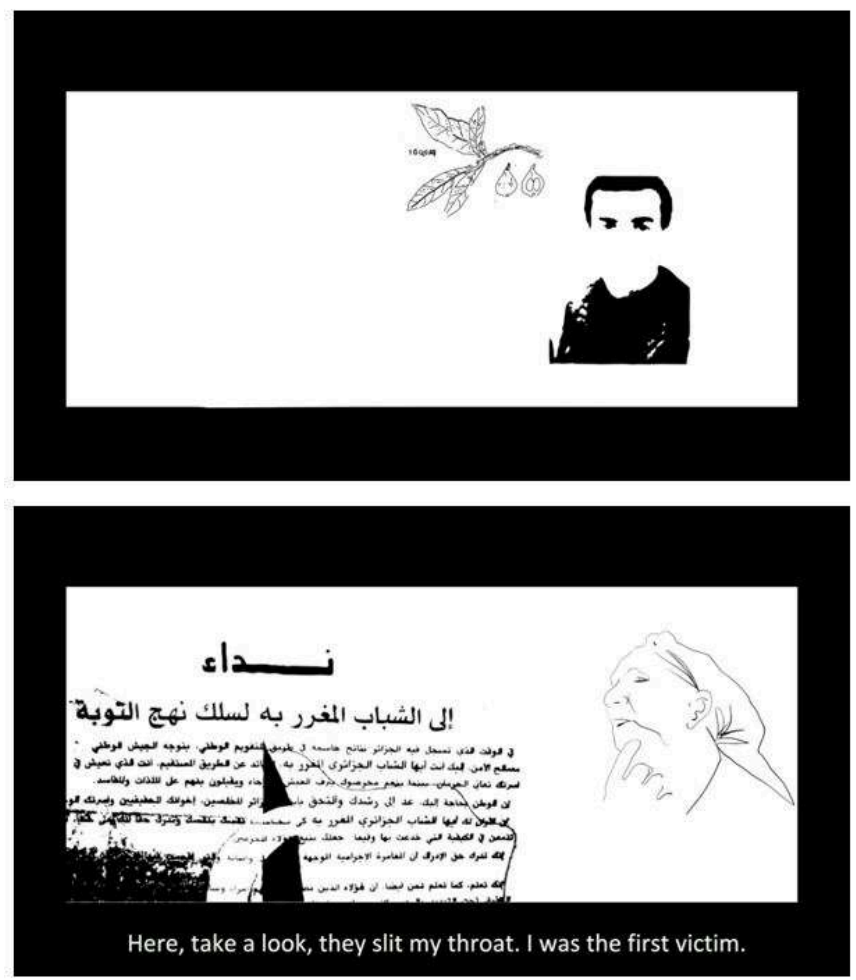

Dessins. Extraits de l'animation What remains (Volet de Memory of Violence).

(c) Sofiane Zouggar

\section{Le Hirak, une archive en train de se faire}

Memory of violence est un projet qui a aujourd'hui sept ans, et les bouleversements que connaît l'Algérie depuis le 22 février 2019 sont une source supplémentaire dans laquelle Sofiane Zouggar va puiser. Il continue de prendre des photos, à saisir les mouvements de foule d'une population qui de manière très visible et très audible investit l'espace public et les villes en particulier. Lorsque je lui demande s'il va inclure les photographies prises lors des manifestations de l'année qui vient de passer, il me répond que oui, certainement. 

partagés. À travers des archives, par un travail d'investigation et de création, Sofiane Zouggar propose de rendre visible ce qui est collectivement considéré comme invisible. Les narrations sur la guerre intérieure manquent, et à travers ce grand projet, l'artiste pallie, à son niveau, le besoin de replacer les images dans leur contexte mais également de les (dé)placer dans un temps de récit car, comme le rappelle John Berger, « le temps du récit devient un temps historique lorsqu'il est assumé par la mémoire et l'action sociales. Le temps du récit fabriqué se doit de respecter le processus de la mémoire qu'il espère stimuler ${ }^{14} »$.

Ce projet au souffle long verra encore la naissance d'un autre volet. Les archives des années 1990, des portraits de disparus de la guerre intérieure par exemple, ont investi les rues en 2019 car elles ont manifestement encore beaucoup de choses à dire, à révéler et à mettre en regard des représentations qui ont jusque-là dominé la sphère visuelle.

deviennent son fonds d'archive personnel, son témo con à lui, sans intermédiaire. Il était jeune pendant les années 1990 et s'il continue à interroger cette période, les nouvelles photographies vont permettre d'explorer, par un prisme nouveau, la guerre intérieure, et de dresser une cartographie de l'expérience, de la mémoire et de la violence.

\section{Conclusion} d'interroger l'expérience de la violence. Il n'est pas anodin que la pratique d'archive se fasse à l'intérieur de la pratique photographique car si l'on peut avoir l'impression qu'une image fixe, arrête les choses, les images, les mouvements et l'histoire, pour les baliser et les circonscrire en quelque sorte, la photographie, elle, saisit. Dans le même mouvement, alors que la période historique des années 1990 s'éloigne, exhumer des archives photographiques et les rendre vivantes au sein d'une pratique artistique, permet de revisiter cette période afin de «nous donner à voir l'histoire, éventuellement notre propre histoire ${ }^{12}$ ».

l'incarnation du rôle de l'intellectuel qui se doit de proposer de manière visible son regard sur le monde, apparaît de manière limpide dans le projet Memory of violence. D'une certaine manière, le travail de recherche de Sofiane Zouggar est celui d'un archiviste résolu à découvrir ce que le récit historique dominant ne lui a pas dit. Il retrouve les archives, les lit et les lie. Il les fait respirer et leur donne une nouvelle place qu'il refuse encore de fixer mais qu'il situe quelque part dans la mémoire de la violence des années 1990.

D'image en image, la mémoire et la violence deviennent des lieux collectivement C'est précisément ce que Sofiane Zouggar propose avec Memory of Violence, par le prisme commun de l'archive et de son art, en croisant son rôle d'artiste et de chercheur, par sa poursuite, déterminée, de la mémoire complexe de sa société.

Sofiane Zouggar (1982) est un artiste multimédia diplômé de l'École supérieure des Beaux-Arts d'Alger. Ses projets sont issus de processus de recherche complexes 
nourris par un travail étroit avec des fonds d'archive publics et privés. Les différents médiums tels que la photographie, la vidéo et le dessin sont autant de potentialités pour ses réflexions artistiques. Artiste-résident de la Villa Romana à Florence en 2013, il est régulièrement invité à participer à des festivals et expositions. Il vit et travaille à Alger. www.sofianezouggar.com.

\section{BIBLIOGRAPHIE}

BERGER John, Understanding a photograph, New York, Aperture, 2013.

FOUCAULT Michel, Le corps utopique, Les hétérotopies, Paris, Nouvelles Éditions Lignes, 2009.

HALL Stuart, "Cultural Identity and Diaspora". J. Rutherford (Ed.), Identity: Community, Culture, Difference London: Lawrence \& Wishart. 1990. p. 222-237.

KRAUSS Rosalind, Le Photographique. Pour une théorie des écarts, Paris, Éditions Macula, (1990), 2013.

LAZALI Karima, le trauma colonial, Paris, Éditions de la Découverte, 2018.

MIRZOEFF Nicholas, “The right to look”, Critical Inquiry, vol. 37, n 3 (printemps 2011), p. 473-496. SAID Edward, Representations of the Intellectual, New York, Vintage Books, 1996.

WESTPHAL Bertrand, La Géocritique, Paris, Éditions de Minuit, 2007.

ZAATARI Akram, « Against Photography: Conversation with Mark Westmoreland », Aperture, $\mathrm{n}^{\circ} 210$, Hello, Photography (printemps 2013), p. 60-65.

\section{NOTES}

1. Stuart Hall, «Cultural Identity and Diaspora », dans Identity: Community, Culture, Difference, J. Rutherford (ed.), Londres, Lawrence \& Wishart, 1990, p. 222-237.

2. Voir Karima Lazali, le trauma colonial, Paris, Éditions de la Découverte. 2018. Lazali parle de guerre intérieure lorsqu'elle se réfère aux violences des années 1990. Jusque-là, il était communément convenu de parler de " guerre civile », de « décennie noire », voire de "décennie rouge ». La difficulté de nommer cette tranche historique est certainement symptomatique du nombre très réduit d'ouvrages (toutes disciplines confondues) qui en traite. Le terme de « guerre intérieure" par sa dimension topographique et psychanalytique me semble être le plus approprié et saisit la complexité et les contradictions auxquelles toute personne qui souhaite travailler sur cette période sera susceptible d'être confrontée.

3. L'expression «Guerre sans image » est popularisée à la suite notamment de la publication de La Guerre invisible. Algérie, années 90, Paris, Presses de la Fondation nationale des sciences politiques, 2001, de l'historien Benjamin Stora.

4. Bertrand Westphal, La Géocritique, Paris, Éditions de Minuit, 2007. 123.

5. Exposition collective, Waiting For Omar Gatlato: Contemporary Art from Algeria and its Diaspora, Wallach Art Gallery, Columbia University, New York, (26 octobre 2019 - 15 mars 2020).

6. La Charte pour la Paix et la réconciliation nationale, votée par référendum en 2005, dans la continuité de la «Concorde civile» de 1999, sanctionne juridiquement la fin de la guerre en accordant l'amnistie aux protagonistes. 
7. À ce sujet, voir Nicholas Mirzoeff, "The right to look», Critical Inquiry, vol. 37, $\mathrm{n}^{\circ} 3$, printemps 2011, p. 473-496, 476. L'auteur résume la série d'opérations de la visualité par trois mouvements.

8. Ibid., p. 473-496, 485, « [...] visuality was a technique for waging war appropriated as a means to justify authority as the imagining of history ».

9. Ibid., p. 473-496, 477. Ma traduction de «Following this direction, the right to look is not simply a matter of assembled visual images but the grounds on which such assemblages can register as meaningful renditions of a given event $»$.

10. Akram Zaatari, «Against Photography: Conversation with Mark Westmoreland », Aperture, $\mathrm{n}^{\circ}$ 210, Hello, Photography (printemps 2013), p. 60-65. Ma traduction de « Against photography also means leaning against photography's history in order to move elsewhere, where we can save photography from its fate ", p. 63.

11. Ibid., p. 60-65, 62. «My relation to photography is mainly one of study; it is a medium I rely on in my art practice; I am an image maker. Having explained how I admire photography's ability to overthrow dominant historical narratives, to present us with multiple histories, even contradictory narratives, I want at the same time to stress that what stimulates me in this study is being able to look at documents with critical distance (both temporal and situational) and being able to compare them with other documents ".

12. Rosalind Krauss, Le Photographique. Pour une théorie des écarts, Paris, Éditions Macula, (1990), 2013, p. 17.

13. Edward Said, Representations of the Intellectual, New York, Vintage Books, 1996.

14. John Berger, Understanding a photograph, New York, Aperture, 2013, p. 54. Ma traduction de "Narrated time becomes historic time when it is assumed by social memory and social action. The constructed narrated time needs to respect the process of memory which it hopes to simulate».

\section{RÉSUMÉS}

C'est par le prisme commun de l'archive et de l'art que l'artiste algérien Sofiane Zouggar s'attèle aux questions de l'image et de la violence de la période des années 1990. Cet article explore les différents volets du projet toujours en cours, Memory of Violence, en interrogeant le geste archivistique de l'artiste dans sa spécificité mais aussi en le rapprochant du geste du chercheur.

It is by the common vantage point of archives and art that the Algerian artist Sofiane Zouggar tackles questions of images and violence during the 1990s. This article explores the different facets of his on-going project Memory of Violence by questioning the artist's gesture of archiving in its specificity but also its proximity to that of the researcher. 
INDEX

Index géographique : Algérie

Mots-clés : Algérie, art, photographie, archive, violence, mémoire

Keywords : photography, archive, violence, memory, Algeria, art

\section{AUTEUR}

\section{RYM KHENE}

RYM KHENE est doctorante en littérature comparée (Thalim- Sorbonne Nouvelle), chercheuse associée à l'équipe Manuscrits Francophones de l'Institut des Textes et Manuscrits (Item, EnsCNRS) et photographe. Ses recherches portent sur les représentations littéraires et photographiques des villes. www.rymkhene.com 